\title{
Experimental two-dimensional field mapping of total internal reflection lateral beam shift in a self-collimated photonic crystal
}

\author{
J. L. Garcia-Pomar, ${ }^{1, a)}$ J. N. Gollub, ${ }^{2}$ J. J. Mock, ${ }^{2}$ D. R. Smith, ${ }^{2}$ and M. Nieto-Vesperinas ${ }^{1, b)}$ \\ ${ }^{1}$ Instituto de Ciencia de Materiales de Madrid, Consejo Superior de Investigaciones Cientificas, \\ Campus de Cantoblanco, Madrid 28049, Spain \\ ${ }^{2}$ Department of Electrical and Computer Engineering and Center for Metamaterials and Integrated \\ Plasmonics, Duke University, P.O. Box 90291, Durham, North Carolina 27708, USA
}

(Received 11 December 2008; accepted 27 January 2009; published online 13 February 2009)

\begin{abstract}
A lateral beam shift is demonstrated both theoretically and in microwave experiments when total internal reflection takes place at the boundary of a self-collimating two-dimensional photonic crystal consisting of an array of high index dielectric cylinders. We further show the dependence of this shift on the cut of the last row of cylinders that defines the crystal interface. (C) 2009 American Institute of Physics. [DOI: 10.1063/1.3085768]
\end{abstract}

It is well known that totally reflected beams at a dielectric-air surface suffer a lateral shift. This is the extensively studied Goos-Hänchen effect, ${ }^{1}$ which is an inherent diffraction effect for an incident beam. The wave components that compose an incident Gaussian beam suffer a nonuniform phase shift upon reflection and contribute to a net offset of the beam. The energy flow between the incident and shifted reflected beams is mediated by evanescent waves on the transmission side (air) of the interface. A similar effect has been discussed in photonic crystals (PCs) when a Gaussian beam impinges from the air with a frequency in the bandgap. $^{2,3}$ In this paper we demonstrate another beam shifting phenomenon that arises in PCs when a guided beam, internal to the crystal, is reflected off a crystal/air interface. It has a certain analogy with the aforementioned Goos-Hänchen ${ }^{1}$ shift, but as we shall see, it occurs at a unique angle of reflection. We remark that a closely related work has recently been reported. ${ }^{4,5}$

Self-waveguiding crystals have been studied in several works both experimentally ${ }^{6}$ and theoretically. ${ }^{7-9}$ Here, we consider a PC configuration consisting of a two-dimensional square lattice of dielectric cylinders. Its waveguiding properties are based on the choice of a particular frequency at which the dispersion isofrequency line is squarelike. ${ }^{8}$ This leads to a collimation effect in privileged directions that happen to be along $\Gamma M$. This is due to the fact that modes inside the PC will propagate with a group velocity given by $v_{g}=\nabla_{k} \omega(k)$, and consequently, the direction of propagation will be perpendicular to the flat portion of the isofrequency contour.

We have built a 2D square PC lattice made of cylinders of flint glass (0080 Corning glass) with permittivity of $\epsilon \approx 7+0.08 i$ in the studied range of frequencies. The PC structure was formed by inserting the glass rods into a matrix of drilled holes in a block Styrofoam material. The refractive index $n$ of Styrofoam is very approximately $n=1$ at these frequencies. The crystal has a lattice constant $a=7.57 \mathrm{~mm}$ and the cylinder radius $r$ is $r=0.35 a=2.65 \mathrm{~mm}$. The crystal is illuminated at a frequency of $\nu=11.76 \mathrm{GHz}$. The sample was introduced in a parallel-plate waveguide, which is com-

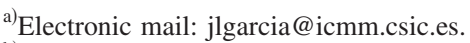

${ }^{b)}$ Electronic mail: mnieto@icmm.csic.es.
}

prised of two flat conducting (Al) plates spaced $11 \mathrm{~mm}$ apart. ${ }^{10}$ Microwaves were introduced through an $X$-band (8 to $12 \mathrm{GHz}$ ) coax-to-waveguide adapter that was attached to the lower plate. A $1.5 \mathrm{~cm}$ wide guide was constructed out of $10 \mathrm{db} / \mathrm{cm}$ absorber along the path from the antenna to the sample to form the incident beam. The sample rested on the lower plate and was nearly of the same height $(10 \mathrm{~mm})$ as the plate separation. A field-sensing antenna was formed from a coaxial fixture inserted into a hole drilled through the upper plate. The center conductor and dielectric of the coaxial connector extended to a position flush with the lower surface of the upper plate and did not protrude into the chamber volume. The lower plate was mounted on two orthogonal linear translation stages so that the lower plate (including the PC prism, waveguide feed, and absorber) could be translated with respect to the upper plate and to the detector. By stepping the lower plate in small increments and recording the field amplitude and phase at every step, a full 2D spatial field map of the microwave scattering pattern could be acquired both inside the sample and in the surrounding free-space region.

We investigated the beam position when total internal reflection takes place at the PC boundary by first numerically simulating a path design based on the experimental setup by Goos and Hänchen ${ }^{1,11}$ which exhibits two total internal reflections, after which the beam propagates outside the PC [cf. Fig 1(a)]. In the experiment we only make one total internal reflection due to the limiting space imposed by the experimental setup [Figs. 1(b) and 1(c)]. We consider a Gaussian incident beam with constant amplitude electric vector $E_{0}$ linearly polarized parallel to the axis of the cylinders and width $\sigma=14 \mathrm{~mm}$, impinging at the bottom surface of the crystal and transmitted within it along the $\Gamma M$ direction. After penetrating, it is self-guided up to the interface where it impinges at an angle of $45^{\circ}$ perpendicular to the $X M$ direction. At this interface, a first total internal reflection is produced along with the appearance of an evanescent wave transmitted into the air. This reflection is repeated a second time at the next interface as illustrated in Fig. 1(a). These reflections bend the beam. This is due to the conservation of the component of the $\vec{k}$ wavevector parallel to the reflection interface. As shown in Fig. 1(d), most of the propagating vectors $\vec{k}_{\mathrm{PC}}$ inside the PC do not provide a permitted propagating 

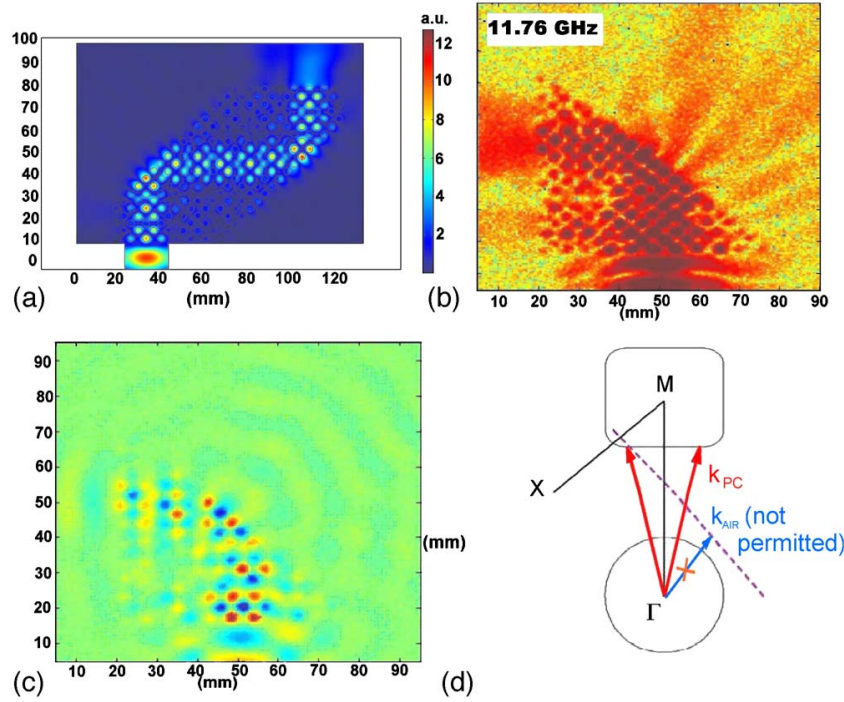

(d)

FIG. 1. (Color online) (a) Map of the modulus of the electric field $|E|$ from a finite elements numerical simulation: an incident Gaussian beam impinges the PC of dielectric cylinders from the bottom and suffers two total internal reflections at the PC boundaries. (b) Experimental data showing the map of intensity with one total internal reflection at an interface parallel to the $X M$ direction in a prism of the same PC of dielectric cylinders, on which the beam enters from below. (c) (movie) Snapshot of the experimental recorded electric field $E_{z}$ showing the field phase in this total internal reflection. (d) Interpretation of the reflection by means of the isofrequency lines.

wavevector $\vec{k}_{\text {air }}$ in the air that satisfies this parallel component conservation. Due to the symmetry of the crystal, only incidence under $\varphi=45^{\circ}$ provides this bending by total internal reflection. When the tilt angle is larger than $45^{\circ}$, there appears a refraction process governed by the Bragg law $k_{\text {air }}^{\|}$ $=K_{G}^{\|}+k_{\mathrm{PC}}^{\|}$, where $K_{G}^{\|}$is the parallel component of the reciprocal lattice vector coming from higher order Brillouin zones. These details are extensively explained in Ref. 8. It is at this unique angle of $\varphi=45^{\circ}$ of the crystal frontier row at which the beam lateral shift occurs and is studied.

To evaluate the reflected beam shift $S$, we measure the position of the beam peak after exiting the crystal, far enough to avoid possible modulations due to the influence of the intensity concentration in the cylinders of the last row. ${ }^{8}$ Another interesting characteristic of this beam shift on total internal reflection in a self-guiding PC is that it stays almost constant (and equal to $2 a$ in this experiment) as the exterior index $n_{2}$ varies (Fig. 2). This behavior may be due to the existence of the unique angle ( $45^{\circ}$ of total reflection) and the existence of a coupling with propagating modes inside the $\mathrm{PC}$, that is, independently of the exterior medium. This implies that another effect is involved in the process of total internal reflection.

Finally, we have investigated the influence of the termination, or truncation, of the cylinders that form the surface of total internal reflection. This truncation has been studied in several works for different geometries with the aim of avoiding losses associated with radiation of surface modes ${ }^{12-14}$ or beaming transmitted light by means of surface modes. ${ }^{15,16}$ We have introduced the cut parameter to define the termination of the crystal [in this case $\tau=r$ corresponds to a hemicylinder and $\tau=2 r=5.3 \mathrm{~mm}$ to the complete cylinder (see inset of Fig. 3)].

Figure 3 shows that the beam shift reaches a maximum about $\tau=1.57 r=4.16 \mathrm{~mm}$, whereas it is minimum for $\tau<r$

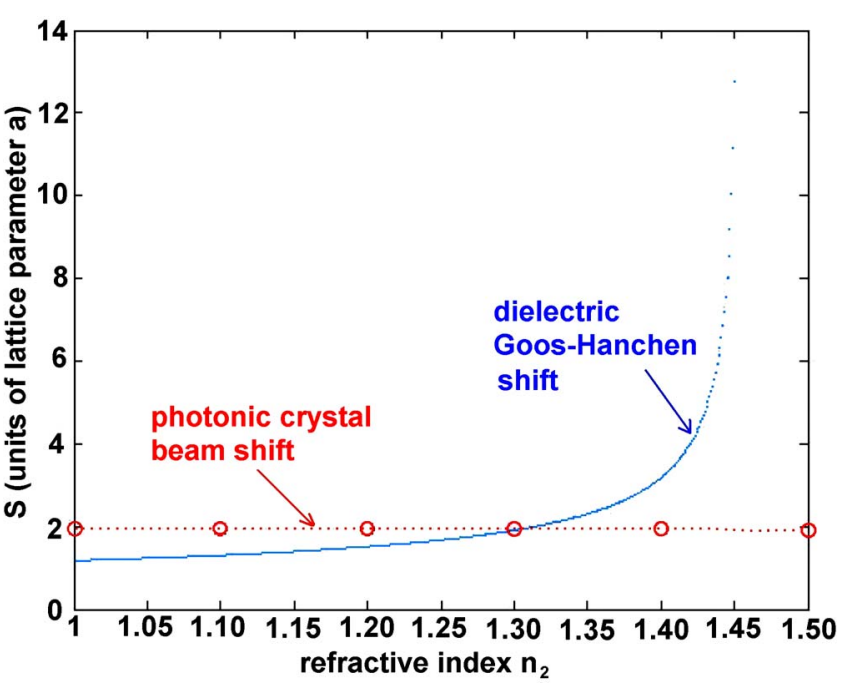

FIG. 2. (Color online) Numerical results of the beam shift $S$ as a function of the exterior refractive index $n_{2}$ for a PC with complete cylinders (red dashed line), in comparison with the beam shift produced by a dielectric (blue solid line) with an effective refractive index similar to the effective index of the PC.

$=2.65 \mathrm{~mm}$. This minimum is due to the existence of the surface modes that exponentially decay on both sides of the interface. These modes coexist inside the PC with propagating modes at the same frequency, and their wavevector $k_{x}$ is smaller than that of the propagating modes (cf. Fig. 4). To demonstrate it, we have employed the supercell method in which slabs of dielectric cylinders alternate with slabs of air. ${ }^{12}$ Therefore, for certain cuts, both the surface modes and propagating modes constructively and destructively interfere, leading to a higher or smaller beam shift, respectively. Possible applications of beam shift were reported before,,${ }^{17,18}$ and a subwavelength concentrator applying this effect was suggested in Ref. 8.

In conclusion, we have characterized a self-collimating flint glass PC showing the dependence of the beam lateral shift on the surface modes by studying the evanescent bands as well as the variation in the exterior refractive index. We also show that the magnitude of this shift is sensitive to the partial truncation of the cylinders of the last row. In this connection, this truncation has an effect similar to the reduction in the last row cylinder radius addressed in Refs. 4 and

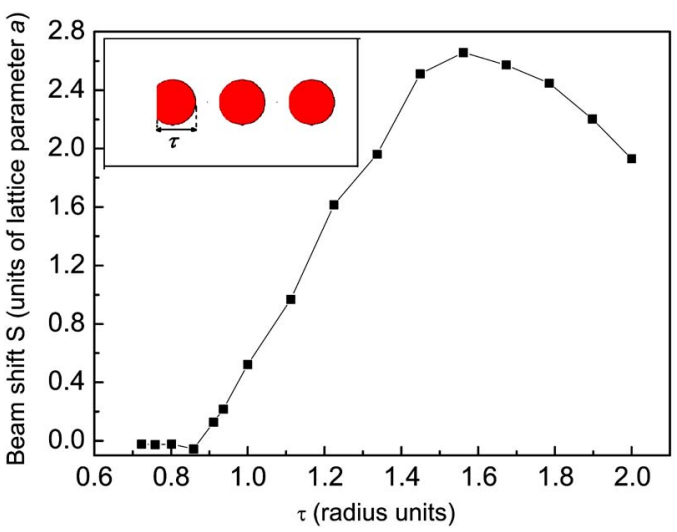

FIG. 3. (Color online) Numerical results of the variation in the beam shift $S$ vs the cut parameter $\tau$ (solid squares) (see inset: truncation in the last cylinder row and representation of the cut parameter $\tau$ ). 


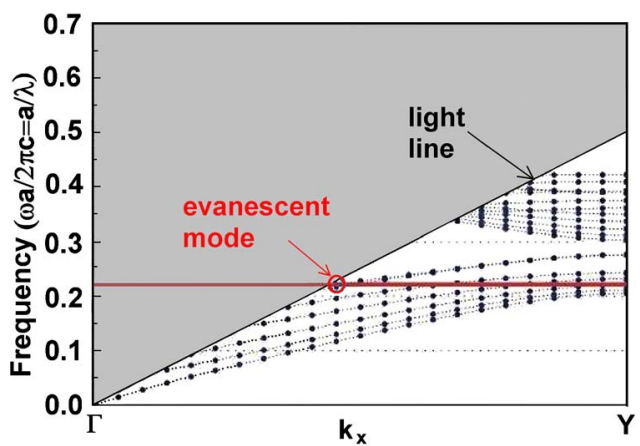

FIG. 4. (Color online) Band diagram for the PC supercell with an evanescent surface mode for a cut $\tau=4.16 \mathrm{~mm}$. The work frequency (solid thick red line) is shown.

5, but at least in these experiments, the truncation seems to reach null beam shift.

The experiment was done at Duke University. J.L.G.P. thanks the support from I3P Grant Program. M.N.V. acknowledges M.E.C. and Consolider Grants of Spain, as well as the European Union. We thank an anonymous reviewer for pointing Refs. 4 and 5 to us.
${ }^{1}$ F. Goos and H. Hänchen, Ann. Phys. 436, 333 (1947); 5, 251 (1949).

${ }^{2}$ D. Felbacq, A. Moreau, and R. Smaâli, Opt. Lett. 28, 1633 (2003).

${ }^{3}$ D. Felbacq and R. Smaâli, Phys. Rev. Lett. 92, 193902 (2004).

${ }^{4}$ A. F. Matthews and Y. S. Kivshar, Phys. Lett. A 372, 3098 (2008).

${ }^{5}$ A. F. Matthews and Y. S. Kivshar, Appl. Phys. Lett. 93, 131901 (2008).

${ }^{6}$ D. Pustai, S. Shi, C. Chen, A. Sharkawy, and D. Prather, Opt. Express 12, 1823 (2004).

${ }^{7}$ X. Yu and S. Fan, Appl. Phys. Lett. 83, 3251 (2003)

${ }^{8}$ J. Garcia-Pomar and M. Nieto-Vesperinas, Opt. Express 13, 7997 (2005).

${ }^{9}$ A. F. Matthews, S. K. Morrison, and Y. S. Kivshar, Opt. Commun. 279, 313 (2007).

${ }^{10}$ B. J. Justice, J. J. Mock, L. Guo, A. Degiron, D. Schurig, and D. R. Smith, Opt. Express 14, 8694 (2006).

${ }^{11}$ K. Iizuka, Elements of Photonics, Volume I: In Free Space and Special Media (Wiley, New York, 2002).

${ }^{12}$ F. Ramos-Mendieta and P. Halevi, Phys. Rev. B 59, 15112 (1999).

${ }^{13}$ W. M. Robertson, G. Arjavalingam, R. D. Meade, K. D. Brommer, A. M. Rappe, and J. D. Joannopoulos, Opt. Lett. 18, 528 (1993).

${ }^{14}$ R. D. Meade, K. D. Brommer, A. M. Rappe, and J. D. Joannopoulos, Phys. Rev. B 44, 10961 (1991).

${ }^{15}$ E. Moreno, F. J. Garcia-Vidal, and L. Martin-Moreno, Phys. Rev. B 69, 121402(R) (2004).

${ }^{16}$ P. Kramper, M. Agio, C. M. Soukoulis, A. Birner, F. Muller, R. B. Wehrspohn, U. Gosele, and V. Sandoghdar, Phys. Rev. Lett. 92, 113903 (2004).

${ }^{17}$ T. Hashimoto and T. Yoshino, Opt. Lett. 14, 913 (1989).

${ }^{18}$ L. Wu, M. Mazilu, T. Karle, and T. F. Krauss, IEEE J. Quantum Electron. 38, 446 (2002). 